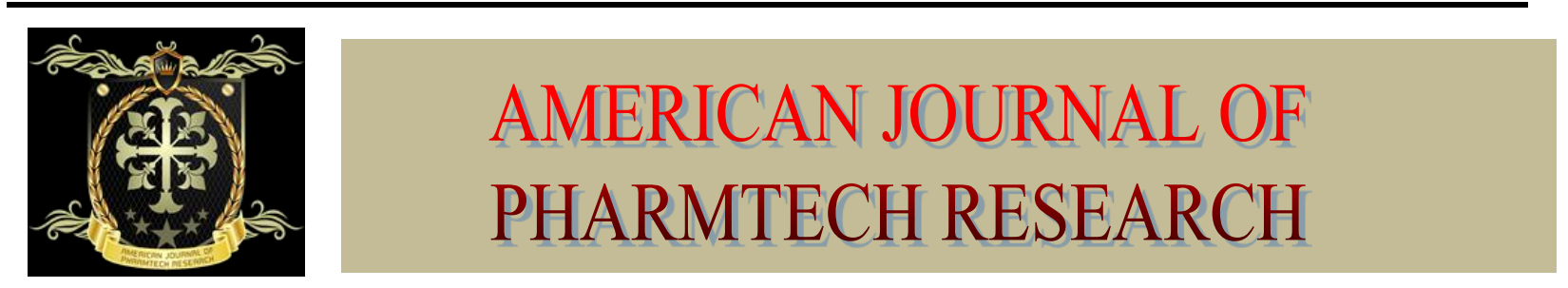

\title{
Preparation and Standardization of Lehya for the Treatment of Asthma
}

\section{Seema Firdouse ${ }^{* 1}$, Zehra Fatima, Tabassum Begum, Asfiya Sultana, Fazeel Sufiyan Dept of Pharmaceutical Analysis and Quality Assurance, Anwarul uloom college of pharmacy, Hyderabad, Telangana}

\section{ABSTRACT}

Lehya is a polyherbal preparation which ensure its action from tongue itself. Our preparation acts by inducing anti inflammatory response on various cells, relaxing smooth muscles resulting in bronchodilation and it also has power to simultaneously enhance memory. Being prepared from plant materials it also has other beneficial effects on health and minimum side effects. The prepared lehya was evaluated for various physiochemical parameters. The preparation was evaluated by TLC and FTIR which have been discuss below. The further scope of the preparation can be studies using GC-MS

Keywords: Lehya, Standardization, TLC, FTIR. 


\section{INTRODUCTION}

Ayurveda is an ancient Indian medical system dating back to the Vedic period of 3000-1500BC. It is considered to be one of the oldest medical health care systems, and is based on sound scientific principles. The word Ayurveda is derived from Sanskrit words ayur and veda which means ayur means life and veda means science ${ }^{[1]}$.

The world health Organization (WHO) defined the herbal drugs as " a finished labeled products that contain active ingredients such as aerial or underground parts of plant or other plant material or combinations of thereof, whether in the crude state or as plant preparations ${ }^{[2]}$.

Herbal medicines are very much in demand because of its safety and efficiency. Medicinal plants are considered as a rich resource of ingredients which can be used in drug development

Pharmacopeial, non-Pharmacopeial or synthetic drugs. The use of herbal treatment is independent of any age group or gender and is in sync with nature ${ }^{[3]}$.

With the ever increasing use of herbal medicines worldwide and rapid expansion of global market of these products, the safety and quality of medicinal plant materials and finished herbal products have become a major concern for public ${ }^{[4]}$ Due to complex nature and inherent variability of chemical constituents of plant based drugs, it is difficult to establish quality control parameters. The pharmacopeia standards for ayurvedic formulations published by government of India, Ministry of health and family welfare give certain physical parameters as standards, however additional methods for quality control based on modern procedures are being developed that can be used as valuable tools in the routine standardization of ayurvedic drugs and formulations ${ }^{[5]}$. Asthma is an inflammatory disorder characterized by airway obstruction, airway hyper responsiveness, and airway inflammation, all of which are variable among patients and variable in time within any specific patient cholinergic mechanisms contribute to increased bronchoconstriction and mucus secretion that limit airflow ${ }^{[6] .}$

The present study is therefore was undertaken to prepare and evaluate lehya for the treatment of asthma. Lehya is a classical Ayurvedic polyherbal formulation explained in Classical text SAHASRAYOGAM 7/10.

\section{MATERIALS AND METHOD}

\section{Procurement of raw materials}

The raw materials were procured from dawasaz, Nampally, Hyderabad, Telangana.

\section{Formula:}

Preparation of Lehya for $100 \mathrm{gm}$ 
Table 1: Formula for preparation of Lehya

\begin{tabular}{lll}
\hline S.no & Crude drug & Quantity for $\mathbf{1 0 0} \mathbf{g m}$ \\
\hline 1. & Aloe Vera & $6 \mathrm{gm}$ \\
2. & Brahmi & $6 \mathrm{gm}$ \\
3. & Tumeric & $1.5 \mathrm{gm}$ \\
4. & Ephedra & $6 \mathrm{gm}$ \\
5. & N. sativa & $1.5 \mathrm{gm}$ \\
6. & Vasaka & $6 \mathrm{gm}$ \\
7. & Ghee & $3 \mathrm{gm}$ \\
8. & Jaggery & $70 \mathrm{gm}$ \\
9. & Methyl Paraben & $0.5 \mathrm{gm}$ \\
& Total & $100 \mathrm{gm}$ \\
\hline
\end{tabular}

\section{Preparation of lehya: ${ }^{[7]}$}

All ingredients in Pharmacopeial quantity were taken. The ingredients Aloe Vera, Brahmi, Turmeric, Ephedra, N. sativa, Vasaka of formulation composition, were washed, cleaned and dried. They were powered and passed through sieve no: 85 to obtained fine powder. Jaggery was taken into S.S Vessel and adds sufficient quantity of water then boiled to dissolve and filtered to muslin cloth and prepares jaggery syrup of thicker consistency by gentle boiling and stirring continuously. Fine powder of ingredients no 1- 6 were added simultaneously when jaggery syrup cooled at room temperature. Ghee was added and levitated that whole product in the end runner mil thoroughly to prepare a homogenous mass. It was stored in containers and packed in air tight containers to protect from light and moisture.

\section{Physiochemical parameters of Lehya: ${ }^{[7]}$}

Macroscopic description and physiochemical analysis, qualitative test, thin layer chromatography and FTIR were conducted. Organoleptic characters like appearance, colour, odour, taste were assessed.

Table 2: Physiochemical parameters of Lehya

\begin{tabular}{lll}
\hline S.No. & Macroscopic Description And Physiochemical Tests & Result \\
\hline 01. & Appearance & Semi solid \\
02. & Colour & Brownish yellow \\
03 & Odour & Pleasant \\
04. & Taste & Sweet \\
05 & Loss on drying & $18 \%$ \\
06 & Total Ash & $3.33 \%$ \\
07 & Acid insoluble ash & $1.66 \%$ \\
08 & Water soluble ash & $41.66 \%$ \\
\hline
\end{tabular}


Table 3: TLC report of Lehya in different solvent systems

\begin{tabular}{lll}
\hline S.No. & Solvent system & Rf value \\
\hline 1 & Benzene : Ethyl acetate & 0.8 \\
2 & Benzene : Diethyl acetate & 0.5 \\
3 & Toulene : Chloroform & 0.5 \\
4 & Butanol : Acetic acid : Water & 0.6 \\
5 & Ethylacetate : Pyridine : Water & 0.4 \\
6 & Benzene : Acetone & 0.7 \\
7 & Chloroform : Pyridine & 0.6 \\
8 & Benzene : Ethanol & 0.7 \\
9 & Chloroform : Acetone : Diethyl amine & 0.9 \\
10 & chloroform : Ethanol & 0.4 \\
\hline
\end{tabular}

Table 4: FTIR range for plant extract

\begin{tabular}{lll}
\hline S No & Range & Functional group \\
\hline 1 & 3874.13 & O-H \\
2 & 3760.04 & O-H \\
3 & 3332.42 & O-H \\
4 & 2561.86 & S-H \\
5 & 2100.71 & C C \\
6 & 1828.00 & C=C \\
7 & 1635.81 & N-H \\
8 & 1507.03 & N-H \\
9 & 1217.40 & S=O \\
\hline
\end{tabular}

Table 5: FTIR range for Lehya

\section{SUMMARY}

\begin{tabular}{lll}
\hline S No & Range & Functional group \\
\hline 1 & 3267.22 & O-H (stretching) \\
2 & 2931.51 & C-C-C, C=C-H \\
3 & 1638.80 & N-H(bending) \\
4 & 1418.70 & C-H \\
5 & 1334.64 & C-O(stretching-alcohols) \\
6 & 1261.97 & C-O(stretching-alcohols) \\
7 & 1044.59 & O-H(bending(alcohols) \\
8 & 988.32 & C-H \\
9 & 923.98 & O-H(bending) \\
10 & 577.87 & C-Br(stretching) \\
11 & 547.68 & C-Br(stretching) \\
\hline
\end{tabular}

Standardization is an important aspect for monitoring and accessing the quality and safety of the poly herbal formulation as these are the combinations of more than one drug to attain the desired therapeutic effect. The Polyherbal formulation has been standardized on the basis of organoleptic properties, physical properties, physio chemical properties TLC and FTIR are used for the identification of formulations well as for the deciding the purity and strength and also for fixing standardization for ayurvedic formulation. The Lehya was evaluated organoleptically as well as 
physio chemically such as color, odour, taste, loss on drying, total ash vales, acid insoluble ash value, acid soluble ash value. The prepared Lehya was semi solid in appearance, brown blackish color, sweetish pungent in taste with characteristic odor. Obtained results of physiochemical parameters was total ash $3.33 \%$, loss on drying $18 \%$, water soluble aash $41.6 \%$., FTIR detected the ranges which revelead the presence of following functional groups i) in the Lehya (3267.22OH(alcohol), 2931.51 C,C=C-H(alkene), 1638.80 N-H(nitro), 1418.70 C-H(alkane), 1334.64CH,1261.97C-O, 1044.59 O-H, 988.32O-H, 923.32C-H, 923.98 O-H, 577.87C-BR,547.68-C-BR) ii) plant extract: $(3874.13$ 0-H, $3760.04 \mathrm{O}-\mathrm{H}, 3332.42 \mathrm{O}-\mathrm{H}, 2561.86 \mathrm{~S}-\mathrm{H}, 2100.71 \mathrm{C}=\mathrm{C}, 1828.00$ $\mathrm{C}=\mathrm{C}, 1635.81 \mathrm{~N}-\mathrm{H}, 1507.3 \mathrm{~N}-\mathrm{H}, 1217.40 \mathrm{~S}=\mathrm{O}$ (sulphur). Major herbal ingredients of prepared medicine have been identified through thin layer chromatography and functional groups was identified through Fourier transform Infrared spectroscopy.

\section{CONCLUSION}

Lehya means the pharmaceutical mode which is ingested in the body by the process of licking. It holds its specialty in many perspectives. The property of licking is very important regarding its mode of action which starts from the tongue itself. The ingredients like Brahmi, Ephedra, N. Sativa and Vasaka have a synergistic effect in several disorders. This pharmaceutical standardization can help and encourage in better understanding of preparation. This process has many pharmaceutical approaches and scopes for the formulation of drug for dreadful ailments.

\section{ACKNOWLEDGEMENT}

I would like to thank DR. Parwez Alam, Associate professor, Department of Pharmacognosy, Shadan College of Pharmacy, Peerancheru, Hyderabad, Telangana for his valuable guidance and support.

\section{REFRENCES}

1. Saraswathi C. research on age of Vedas, the Vedas, (Bhartiya vidya bhavan, Bombay) 1988,16-20).

2. Ayuverdic formulary of India, part $1,2^{\text {nd }}$ revised English Edison,(Govt of India, Ministry of health and family welfare; New Delhi), 2003, 33-34

3. WHO guidelines for assessing quality of herbal medicines with reference to contaminants and residues by World health organization 2007;01

4. Gupta mansi, Int journal of traditional knowledge, vol 10(4) oct 2011 pp.594-598.

5. Adv Exp Med Biol. 2014;795:5-15. doi: 10.1007/978-1-4614-8603-9_1Department of Internal Medicine, University of Texas Medical Branch, 4.118 John Sealy Annex, 301 University Blvd, Galveston, TX, 77555-0568, USA.) 
6. Bhaishajya Kalpanaa - The Ayurvedic Pharmaceutics - An Overview SS Savrikar Afr J Tradit Complement Altern Med. 2010; 7(3): 174-184. Published online 2010 Apr 3. PMCID: PMC3025621; PMID: 21461144

7. Dr. Priyanka Triwedi* Pharmaceutical Standardization Of Ashwagandhadilehya ;; Research Research Article ISSN 2394-3211 EJPMR.

\section{AJPTR is}

- Peer-reviewed

- bimonthly

- Rapid publication

Submit your manuscript at: editor@ajptr.com

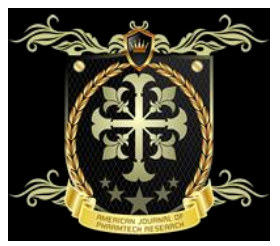

